\title{
Comparative genomic analysis of the Hafnia genus reveals an explicit evolutionary relationship between the species alvei and paralvei and provides insights into pathogenicity
}

\author{
Zhiqiu Yin ${ }^{1,2,3 \dagger}$, Chao Yuan ${ }^{1,2,3 \dagger}$, Yuhui Du ${ }^{1,2,3 \dagger}$, Pan Yang ${ }^{1,2,3}$, Chengqian Qian ${ }^{1,2,3}$, Yi Wei $i^{1,2,3}$, Si Zhang ${ }^{1,2,3}$, \\ Di Huang ${ }^{1,2,3^{*}}$ and Bin Liu ${ }^{1,2,3^{*}}$ (D)
}

\begin{abstract}
Background: The Hafnia genus is an opportunistic pathogen that has been implicated in both nosocomial and community-acquired infections. Although Hafnia is fairly often isolated from clinical material, its taxonomy has remained an unsolved riddle, and the involvement and importance of Hafnia in human disease is also uncertain. Here, we used comparative genomic analysis to define the taxonomy of Hafnia, identify species-specific genes that may be the result of ecological and pathogenic specialization, and reveal virulence-related genetic profiles that may contribute to pathogenesis.

Results: One complete genome sequence and 19 draft genome sequences for Hafnia strains were generated and combined with 27 publicly available genomes. We provided high-resolution typing methods by constructing phylogeny and population structure based on single-copy core genes in combination with whole genome average nucleotide identity to identify two distant Hafnia species (alvei and paralvei) and one mislabeled strain. The open pan-genome and the presence of numerous mobile genetic elements reveal that Hafnia has undergone massive gene rearrangements. Presence of species-specific core genomes associated with metabolism and transport suggests the putative niche differentiation between alvei and paralvei. We also identified possession of diverse virulence-related profiles in both Hafnia species., including the macromolecular secretion system, virulence, and antimicrobial resistance. In the macromolecular system, T1SS, Flagellum 1, Tad pilus and T6SS-1 were conserved in Hafnia, whereas T4SS, T5SS, and other T6SSs exhibited the evolution of diversity. The virulence factors in Hafnia are related to adherence, toxin, iron uptake, stress adaptation, and efflux pump. The identified resistance genes are associated with aminoglycoside, beta-lactam, bacitracin, cationic antimicrobial peptide, fluoroquinolone, and rifampin. These virulence-related profiles identified at the genomic level provide insights into Hafnia pathogenesis and the differentiation between alvei and paralvei.
\end{abstract}

(Continued on next page)

* Correspondence: huangdi@nankai.edu.cn; liubin1981@nankai.edu.cn

†Zhiqiu Yin, Chao Yuan and Yuhui Du contributed equally to this work.

'Key Laboratory of Molecular Microbiology and Technology of the Ministry

of Education, TEDA College, Nankai University, Tianjin, People's Republic of

China

Full list of author information is available at the end of the article

(c) The Author(s). 2019 Open Access This article is distributed under the terms of the Creative Commons Attribution 4.0 International License (http://creativecommons.org/licenses/by/4.0/), which permits unrestricted use, distribution, and reproduction in any medium, provided you give appropriate credit to the original author(s) and the source, provide a link to the Creative Commons license, and indicate if changes were made. The Creative Commons Public Domain Dedication waiver (http://creativecommons.org/publicdomain/zero/1.0/) applies to the data made available in this article, unless otherwise stated. 
(Continued from previous page)

Conclusions: Our research using core genome phylogeny and comparative genomics analysis of a larger collection of strains provides a comprehensive view of the taxonomy and species-specific traits between Hafnia species. Deciphering the genome of Hafnia strains possessing a reservoir of macromolecular secretion systems, virulence factors, and resistance genes related to pathogenicity may provide insights into addressing its numerous infections and devising strategies to combat the pathogen.

Keywords: Hafnia, Comparative genomics, Macromolecular secretion system, Virulence factors, Antibiotic resistance

\section{Background}

Hafnia is a common genus of gram-negative bacteria found in the environment on plants and foods as well as in the gastrointestinal tracts of mammals. This genus belongs to the Enterobacteriaceae family and includes two known species (alvei and paralvei) [1]. Since its discovery more than 50 years ago, very little has been determined about variations among Hafnia species. Previous investigations of the taxonomy of Hafnia were based on 16S rRNA, DNA-DNA hybridizations and multilocus enzyme electrophoresis [1, 2]. In addition, many traditional phenotypic characteristics have failed to distinguish these two species from each other $[1,3]$. The low cost of next-generation sequencing has generated an upsurge in microbial genome sequences that has allowed researchers to characterize microorganisms using genome data. Therefore, whole-genome sequencing data provide the opportunity to re-evaluate current Hafnia species definitions and establish an accurate species definition for the taxonomically challenging genus Hafnia.

There are no comprehensive genome-wide analyses of the taxonomy of the Hafnia genus. To better understand the phylogenetic relationship and genomic distances that distinguish distinct species, we generated genome sequence data for 20 Hafnia strains with different $\mathrm{O}$-antigens and performed phylogeny, population genetic structure and whole genome average nucleotide identity analyses with other publicly available Hafnia sequences. To expand the genomic perspective with regard to inter-species diversity and differences, we further constructed core and pan-genome analyses and characterized the species-specific core genome that provides insights into the divergence and niches differentiation between alvei and paralvei.

Members of the Hafnia genus are commonly isolated from the gastrointestinal tract of humans and animals and from foods [4]. Hafnia, as opportunistic pathogens, may cause acute gastroenteritis [2]. Notably, it has more commonly been reported to cause severe extra-intestinal disease, including bactereamia and respiratory tract infections, primarily in immunocompromised patients, patients with penetrating soft tissue injury, and organ transplant patients $[5,6]$. Unfortunately, very little is known about these strains in regard to their role as pathogens. Benefiting from the increasing feasibility of next-generation sequencing and the advent of bioinformatics tools during recent years, we focused on analysing and defining the virulence-related genetic profiles of Hafnia within a large number of genomes to reveal its pathogenic potential.

The use of whole genome sequences has been regarded as a promising avenue for taxonomic and phylogenetic studies of Hafnia and for virulence and resistance genotypic profiles of species alvei and paralvei. In the present study, we generated one complete genome sequence and 19 draft genome sequences for Hafnia. These sequences were supplemented by 27 additional genomes of Hafnia strains available in public databases. The implementation of our study is divided into three parts: (i) we perform phylogenetic and population structure analysis based on single-copy core genes in combination with average nucleotide identity to elucidate the taxonomy of Hafnia; (ii) core and pangenome analysis of alvei and paralvei define the species-specific core genome and reveal the interspecies genomic difference, thus expanding our understanding of divergent evolution and adaptation to diverse niches and providing a simple and fast approach for identifying distinct species of Hafnia; and (iii) we identify the profiles related to macromolecular secretion systems, virulence, and antimicrobial resistance. The identification of these genomic features will provide further insight into the evolution and pathogenic potential of Hafnia.

\section{Results}

Whole-genome based phylogeny, population genetic analysis, and average nucleotide identity provided a highresolution taxonomy

To assess phylogenetic relationship of the Hafnia genus, a phylogenetic tree was constructed using the concatenated nucleotide sequence of 2045 core genes (Additional file 2: Table S2) from 20 newly sequenced and 27 publicly available Hafnia strains. The core genome tree generated a reliable delineation of phylogenetic relationships across the Hafnia genus. According to our core genome tree, the 47 strains were divided 
into two phylogenetic lineages (Fig. 1); one lineage contained 26 strains (designated alvei), while the other lineage included 21 strains (designated paralvei). The lineages alvei and paralvei formed distinct, extremely tight clusters in separate clades from each other, suggesting that the genetic differentiation between the genomes of alvei and paralvei strains occurred during adaptation to different niches, and co-evolution with their hosts, in which other microbes could be an important driving factor. To further explore the genomic similarities among strains, we complemented our phylogeny with genetic population structure analysis using the program STRUCTURE [7]. As shown in Fig. 1, the strains generally clustered into two structure clusters in a pattern matching that observed in the phylogenetic analysis. Our phylogenetic analysis and population structure exhibited a reliable delineation of genetic relationships between the species alvei and paralvei. In addition, we identified a mislabeled strain, ATCC 51873, which was previously classified as the species alvei and should be corrected to paralvei.

The average nucleotide identity (ANI) value was applied to delineate species and was calculated to estimate the genetic distance between strains at the genomic level [8]. Here we calculated the pairwise ANI values of 47 strains to examine the inter-species genetic relatedness within the Hafnia genus. Based on the ANI results, the strains were clearly divided into two groups, which was consistent with the phylogenetic analysis results. The ANI values for alvei and paralvei were above 96 and 93\% (Additional file 3: Table S3), respectively. It is worth noting that the ANI values between alvei and paralvei were approximately $87 \%$, below the recommended $95 \%$ threshold value for species circumscription [8], illustrating the prominent genetic distance between these two species.

\section{Characterizing the core and pan-genomes}

To assess the genetic diversity, we constructed the core (genes shared among all 47 strains) and pan (all genes found across all 47 strains) genome curves of the Hafnia genus (Fig. 2a). In our pan-genome of Hafnia, 13,255 gene families were identified across 47 genomes, of which 2529 constitute the core genome. The pan genome curve is noticeably shaped by the number of novel gene additions with each additional genome. Conversely, a continuous decline in the core genome curve was observed for the novel additional genome. Interestingly, the pan-genome curve was strongly affected by the addition of a large number of novel gene families in additional alvei genomes (Fig. 2a), suggesting that there are substantial differences in gene content between alvei and paralvei.

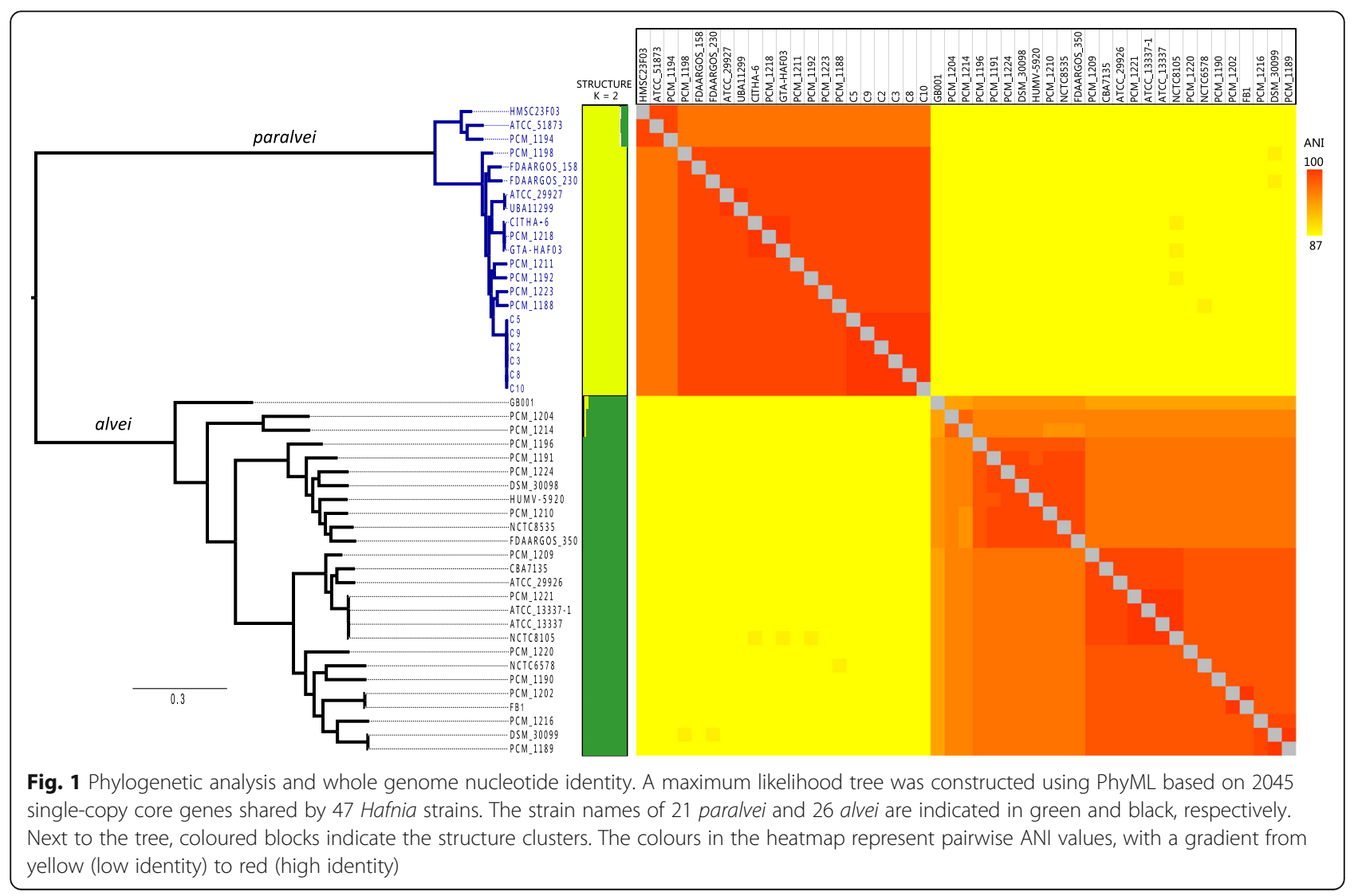




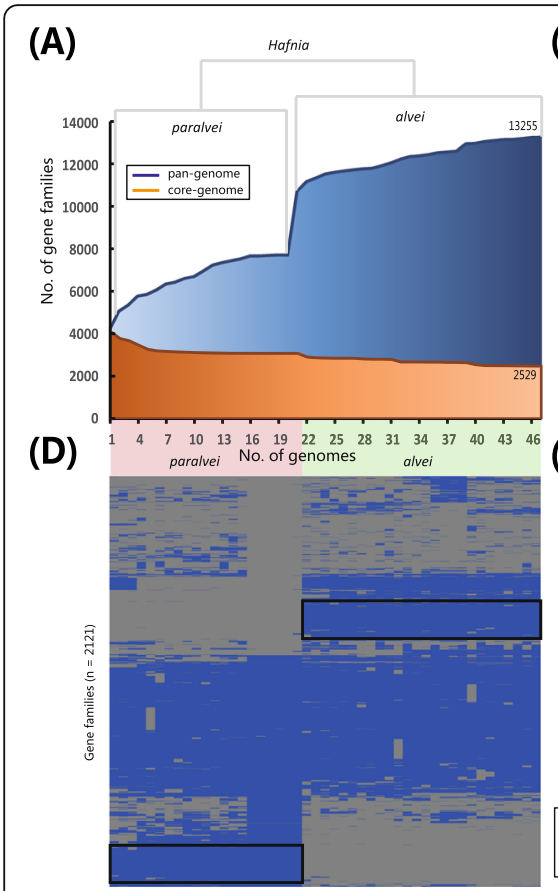

(B)
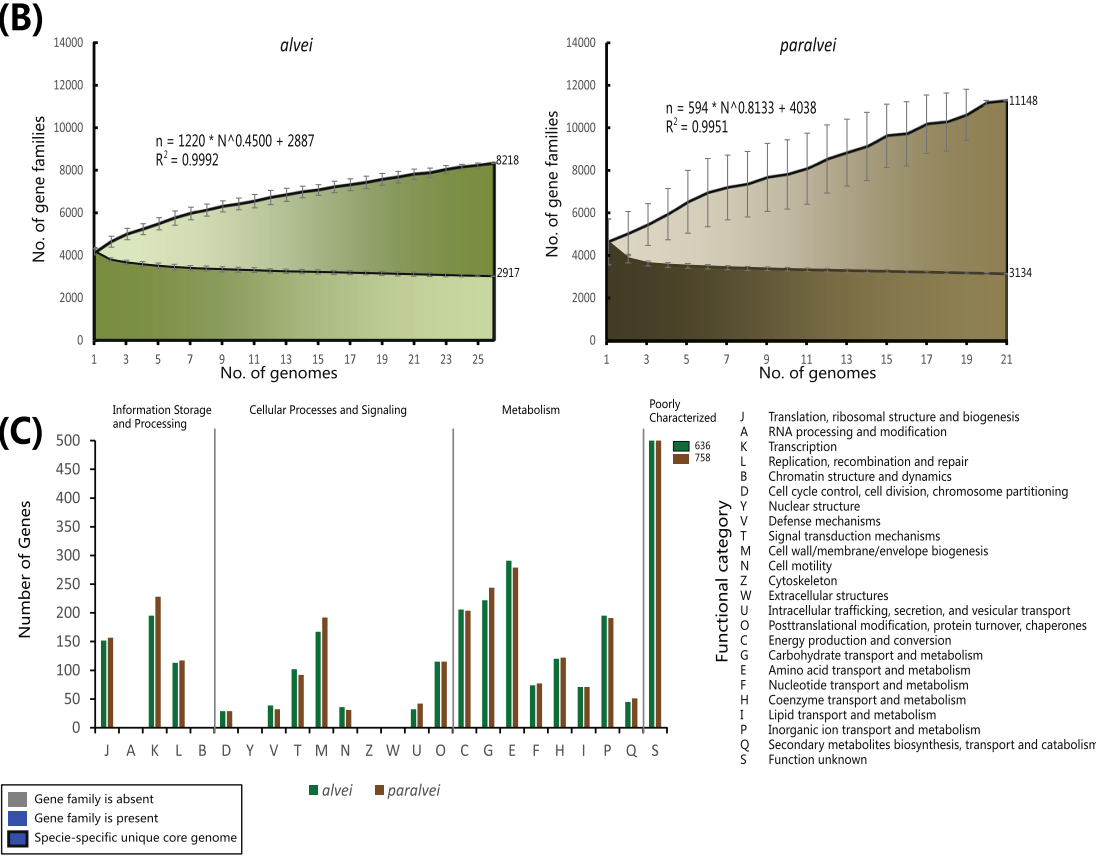

Fig. 2 Core and pan-genome analysis of Hafnia. a The Hafnia core and pan-genome were constructed for 47 genome sequences of Hafnia, representing 2 species: 21 paralvei and 26 alvei. The genomes are in the same order as in the core genome tree (Fig. 1). b Core and pan-genome curves for genome sequences of alvei and paralvei show the downward trend of the core gene families and the upward trend of the pan gene families with the increasing number of genomes. We show the average and standard deviation of each value. The deduced mathematical function of pan-genome curves was reported. c Distribution of functional catalogues of alvei and paralvei. $\mathbf{d}$ Cluster map of the accessory genome of alvei and paralvei. The gene families that are unique to a species and conserved across most strains in that species are framed in black

Having determined the genomic differences, we characterized the core and pan-genomes of alvei and paralvei separately (Fig. 2b). The pan-genomes of both species show a clear linear upward trend in agreement with the Heap's law pan-genome model [9], and a robust fit to the data of both species was obtained with an increasing power law with positive exponents of $\gamma=0.4500$ (alvei) and 0.8133 (paralvei). (Fig. 2b). The exponent $\gamma>$ 0 indicates an open pan genome species [9]. Additionally, we found that the paralvei pan-genome is approximately 3000 genes larger than the alvei pan-genome (11, 148 and 8218, respectively), suggesting that strains of this species have more frequent genetic exchange events and a large source of gene content.

Compared to the pan-genome curves, the number of gene families decreased sharply with additional genomes, reaching minimum values of 3134 and 2917 for alvei and paralvei, respectively. We further used the Cluster of Orthologous Group (COG) assignments to determine the functional categories of the core gene families of alvei and paralvei. The core gene families of both species were unevenly distributed across the functional categories (Fig. 2c). Larger proportions of the core gene families of both alvei and paralvei were involved in the transcription (category K: 6.8 and 7.5\%), energy production (category C: 7.3 and 6.7\%), transport and metabolism of carbohydrates, amino acids and inorganic ion (categories G: 7.8 and 8.0\%, E: 10.2 and 9.2\%, and P: 6.8 and $6.3 \%$ ). It is notable that most of the core gene families in both alvei and paralvei play important roles in maintaining growth and reproduction.

\section{Species-specific core genomes revealed the extent of divergence between alvei and paralvei}

The species-specific pan-genome content reveals that these are an underlying profiles of gene families that are conserved among strains of a species, some of which are unique to this species. To identify the species-specific gene families, we constructed the accessory genome by subtracting the core genome and low frequency genes ( $<10$ examples) from the pangenome. As shown in Fig. 2d, the cluster map of the Hafnia accessory genome demonstrates that each serovar is differentiated by a set of conserved gene families (framed in black, Fig. 2d). A total of 213 and 183 gene families were identified as part of the alvei and paralvei-specific core genomes (Additional file 4: Table S4), respectively. Bared on KEGG annotation, the functional categories "carbohydrate metabolism", "lipid metabolism", "metabolism of cofactors and vitamins" and 
"membrane transport" were enriched in the alvei-specific core genome; the functional categories "carbohydrate metabolism" and "membrane transport" were enriched in the paralvei-specific core genome (Fig. 3a). These speciesspecific genes indicate the putative niche differentiation between alvei and paralvei.

Three and two complete pathway modules are present in the alvei and paralvei-specific core genomes (Fig. 3b), respectively. The EvgS-EvgA two-component regulation system in Escherichia coli is a transcriptional regulator of drug efflux genes and closely is related to multi-drug resistance [10]. The cellobiose phosphotransferase system confers the assimilation of cellobiose by cleaving the disaccharide into glucose and glucose-1-phosphate, which serve as carbon and energy sources [11, 12]. MetD (MetI-N-Q) is a high-affinity transport system for methionine, which is an important amino acid involved in numerous metabolic processes in bacteria [13]. The

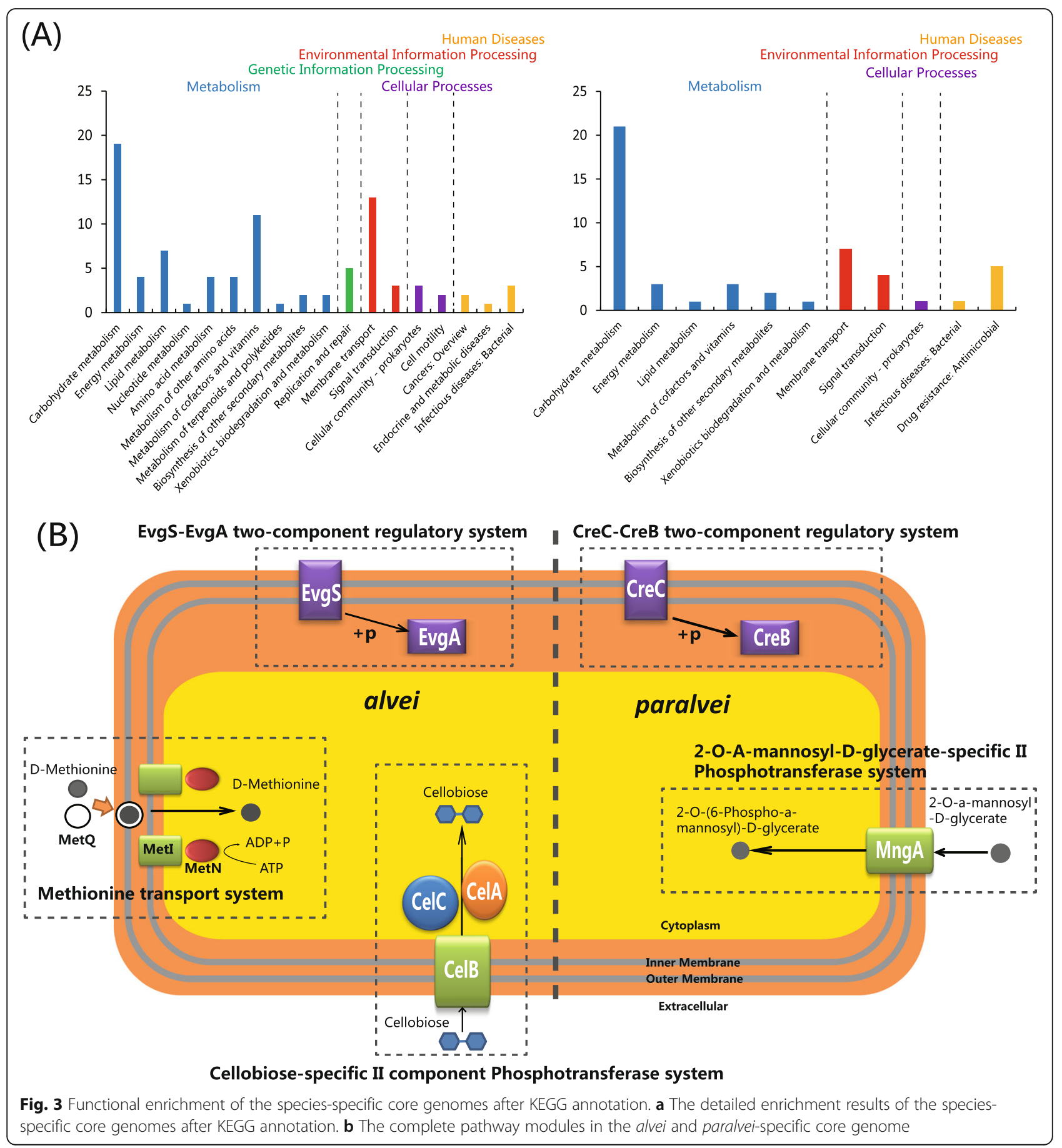


CreC-CreB (carbon source-responsive) two-component regulation system in Escherichia coli affects a number of functions, including the intermediary carbon catabolism and intracellular redox state [14]. 2-O- $\alpha$-mannosyl-D-glycerate is taken up by the MngA phosphotransferase system and utilized as a sole carbon source [15]. Our analysis revealed that genes related to metabolic pathways, antimicrobial resistance and virulence were part of these unique species-specific core genomes, which helped us to characterize the genomic differences between alvei and paralvei. These species-specific genes might serve as a mark for distinguishing alvei and paralvei.

\section{Macromolecular secretion systems reflected the pathogentic potential of Hafnia and Mobile genetic elements mediated the genomic plasticity}

Secretion is an essential task for bacteria to interact with their surrounding environment [16]. In particular, many virulence factors in pathogens are secreted [17]. The production of extracellular proteins is important for many aspects of bacterial competition and adaptation such as virulence, antimicrobial resistance, detoxification and scavenging [18]. In gram-negative bacteria, six types of secretion systems (T1SS to T6SS) have been identified and well characterized by numerous experimental studies [19-21]. Here, we identify occurrences of the macromolecular secretion systems in 47 Hafnia genomes using MacSyFinder [22]. The model and evolution of macromolecular secretion systems identified in Hafnia genomes are described in Figs. 4, 5, and 6. In the following sections we describe the models of each type of macromolecular secretion system.

Gene clusters of types I, III, IV, V, VI and homologues of the flagellum, and Tad pilus secretion systems, were found in the Hafnia genomes (Fig. 4). Although T1SS, Flagellum 1, Tad pilus, and T6SS-1 are restricted to Hafnia, T3SS, T4SS, T5SS, and other T6SSs are not wholly exclusive to strains of alvei and/or paralvei. These strain-specific secretion systems might be horizontally transferred from other species. Furthermore, numerous mobile genetic elements (MGEs) included clustered regularly interspaced short palindromic repeats (CRISPRs), insertion sequences (ISs), genomic islands, and prophages that have been identified across the study genomes (Fig. 4). These elements are the

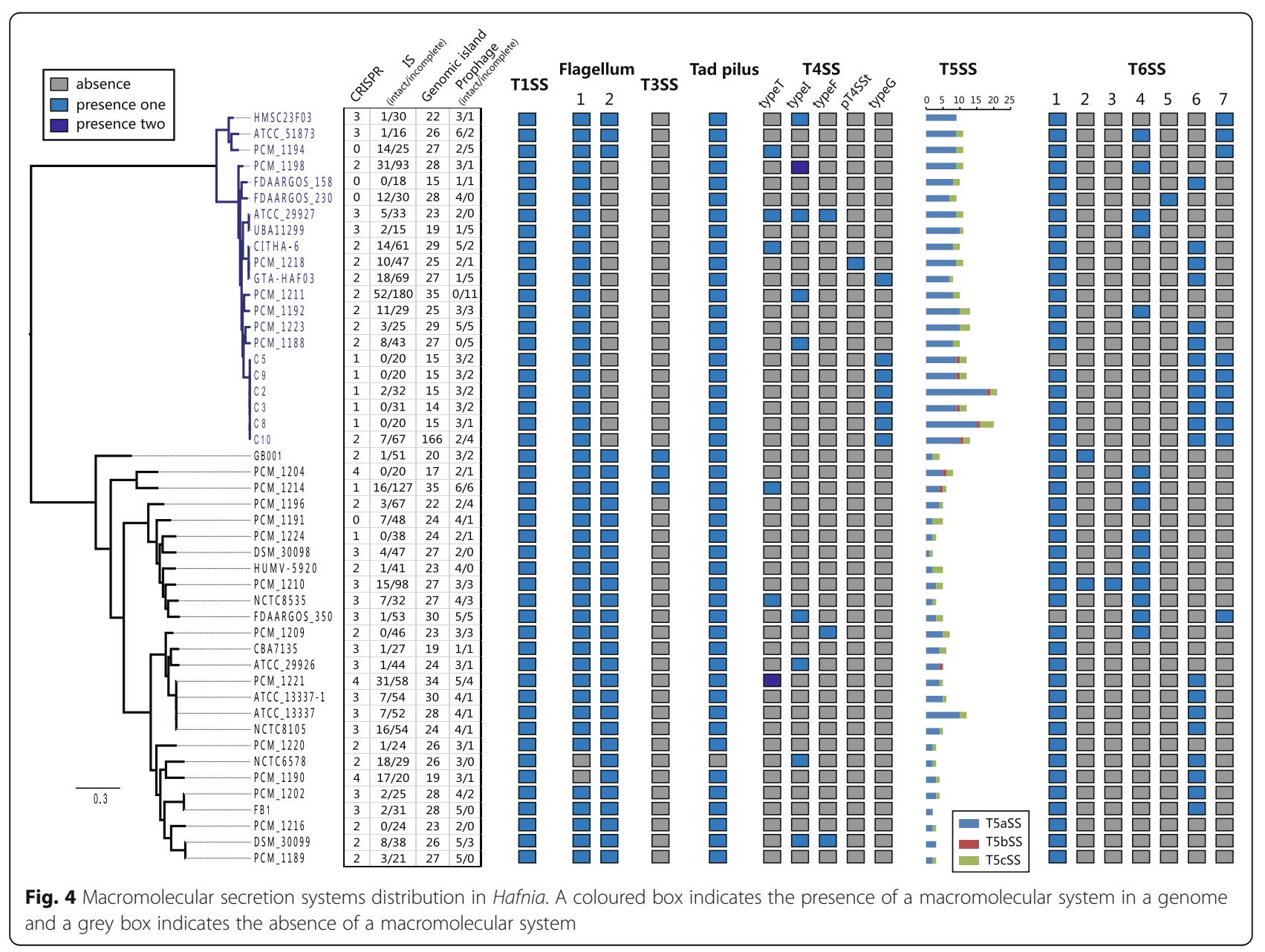




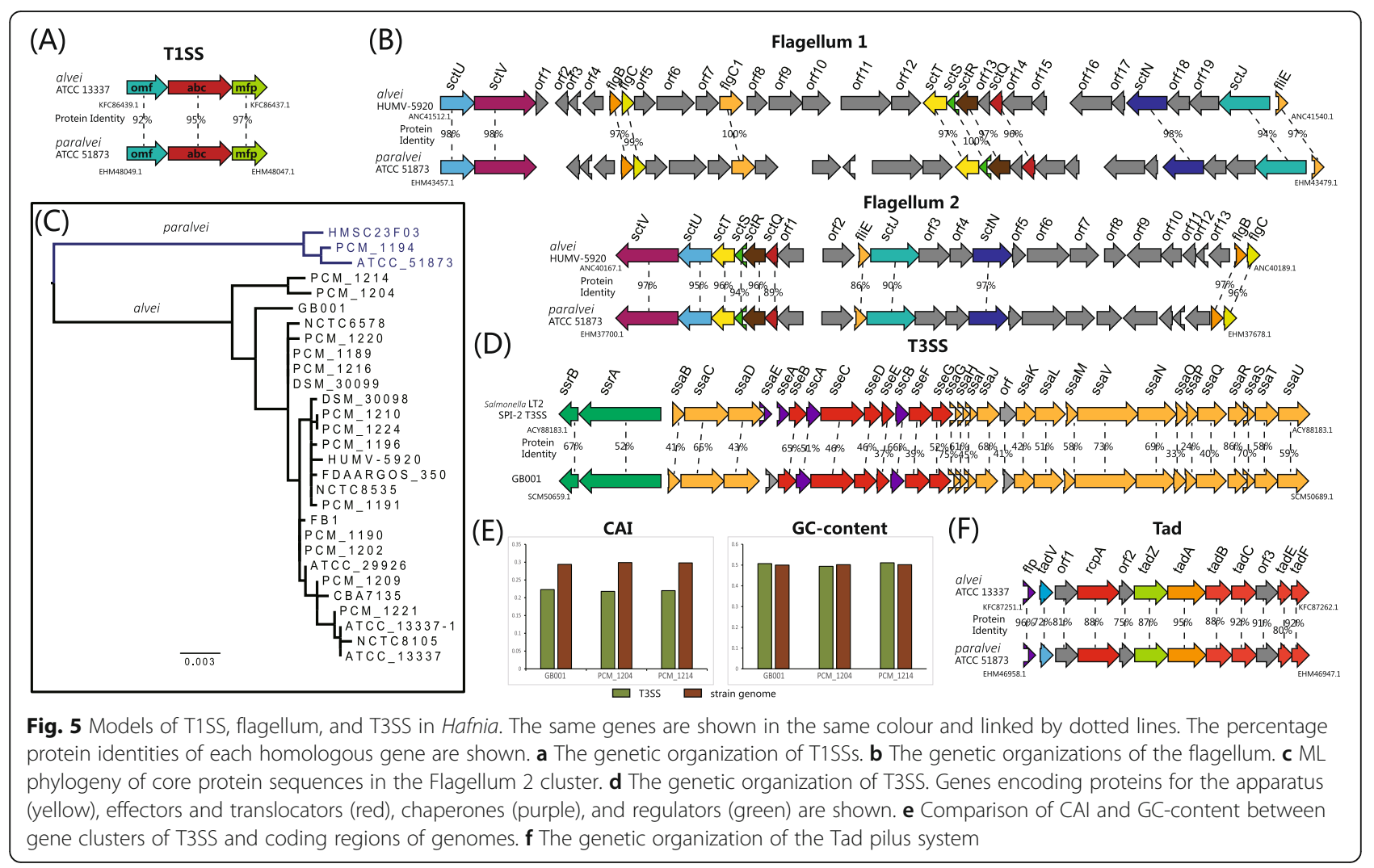

major contributors to horizontal gene transfer (HGT), and drive the adaptation of bacteria to diverse niches. The diverse secretion systems may be acquired as part of the MGEs.

\section{Conserved the type I secretion system}

T1SS is composed of three indispensable membrane proteins, an $\mathrm{ABC}$ transporter ( $\mathrm{ABC}$ : providing an inner membrane channel), a membrane porin (OMF: forming the outer membrane channel) and an inner membrane anchored adaptor protein (MFP: connecting the OMF and the $\mathrm{ABC}$ components) [23]. T1SS can secrete many proteins, including haemolysins for pathogenesis in the host organism, and some extracellular proteases for nutrient acquisition, some bacteriocins for antibacterial activity $[18,20,24]$. We found that the T1SS cluster was

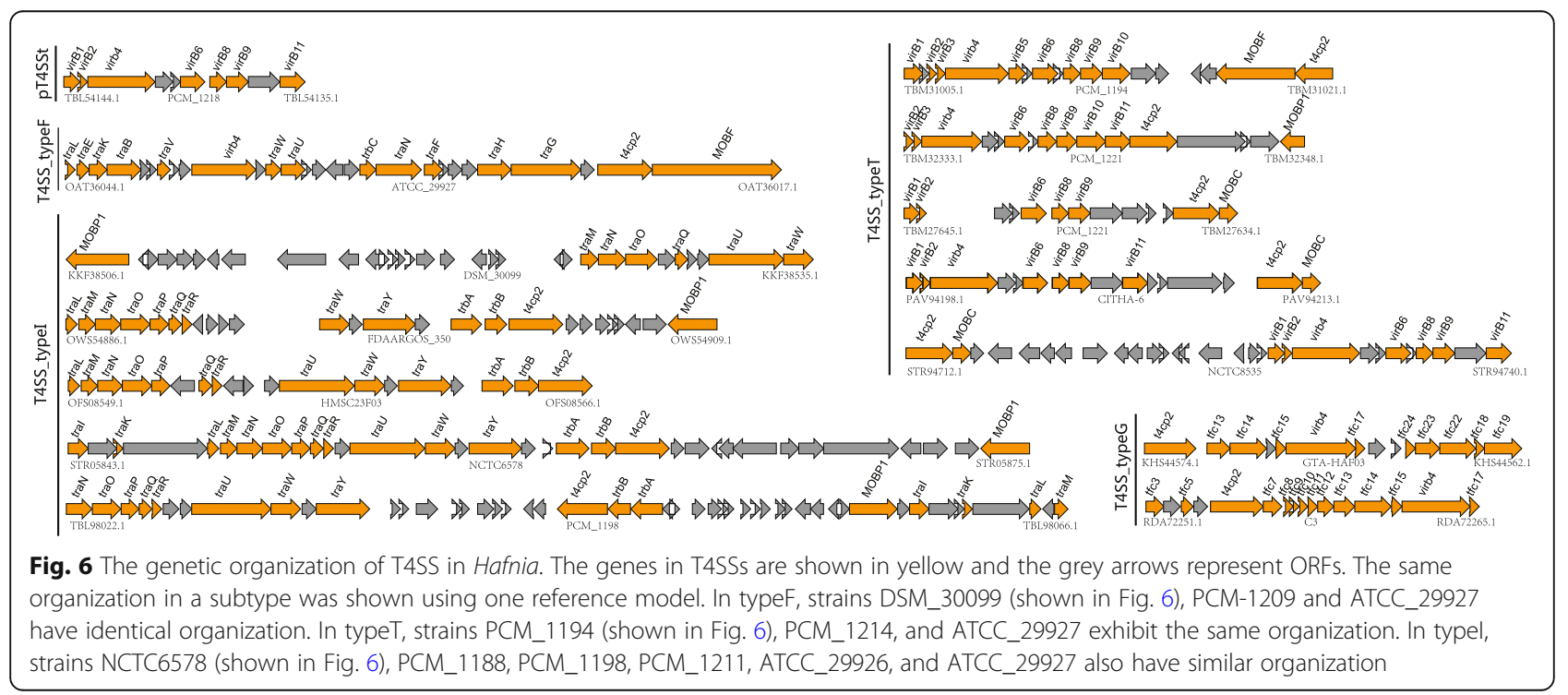


present in all 47 Hafnia genomes. Both alvei and paralvei shared a common T1SS cluster (Fig. 5a). The core components $\mathrm{ABC}$, OMF and MFP were encoded together and highly conserved (protein identity >92, Fig. 5a), thus indicating that this T1SS is restricted and conserved in the Hafnia genus.

\section{Conserved and species-specific flagellum and incidental the type III secretion system}

The flagellum and T3SS are two of the most impressively large macromolecular complexes spanning both membranes of gram-negative bacteria [25]. Two types of flagellum systems are identified in Hafnia genomes (designated Flagellum 1 and Flagellum 2, Fig. 5c). Flagellum 1 is present in both alvei and paralvei except for two alvei strains (NCTC 6578 and PCM 1190). As shown in Fig. 4, Flagellum 2 is present in all alvei strains and only three paralvei strains (HMSC23F03,ATCC 51873, and PCM 1194). Furthermore, we performed phylogenetic analysis using the core genes of Flagellum 2 (Fig. 5d), and the resulting tree revealed a similar topology to that of the core genome tree (Fig. 1). Our analysis shows that most strains of paralvei do not contain Flagellum 2 due to an earlier deletion event.

T3SS evolved from the flagellum and is at the centre of the export machinery that enables the direct transfer of proteins from the bacterial cytosol into the host cells [25]. T3SSs are usually encoded in a single locus, and many are homologous to components of the flagellar apparatus [26]. Three alvei genomes (GB001, PCM 1204, PCM 1214) had a complete set of T3SS genes (Fig. 5d). We compared the CAI (codon adaptation index) and GC-content between the T3SS gene clusters and three host genomes. The T3SS gene clusters displayed an apparent deviation in CAI (Fig. 5e), and it is likely that these T3SS gene clusters were acquired by HGT events. Furthermore we identified the putative homologous T3SS using blastp searches of the NCBI non-redundant protein database. We found that the well-studied T3SS of Salmonella pathogenicity island 2 (SPI-2) was closely related to the T3SS of Hafnia, and they inhabit similar gene loci and show approximately $54 \%$ identity between protein sequences(Fig. 5d). The function of the T3SS encoded by SPI-2 is central to the ability of S. enterica to cause systemic infections and for intracellular pathogenesis $[27,28]$. It is worth noting that these three alvei strains with T3SS have the potential to cause systemic disease.

\section{Conserved the tight adherence pilus}

The Tad pilus secretion system plays a role in biofilm formation, pathogenesis, adhesion or natural transformation in many bacteria [29, 30]. In the Hafnia genus, a Tad pilus system has been identified in all but one alvei strain (NCTC 6578). The components of the Tad systems of both alvei and paralvei are encoded within one genetic cluster and share identical genetic organization and high identity of protein sequences (Fig. 5f). Therefore, our results reveal that this Tad pilus system is restricted and conserved in the Hafnia genus.

\section{Strains-specific the type IV secretion system}

T4SSs transport a diverse array of substrates, including DNA, DNA-protein complex, and proteins into the host cells, and play fundamental roles in both pathogenesis and adaptation in the host cellular niche. T4SSs are divided into eight subtypes [16, 31]. Our analysis revealed that 23 Hafnia genomes possessed T4SSs comprised of types T, I, F, G, and pT4SSt. The strain PCM_1218 possesses pT4SSt, which is a secreted protein system, and other T4SSs in Hafnia were the conjugationrelated T4SSs. The number of genes in Hafnia T4SSs ranges from 7 to 15 . As shown in Fig. 6, the genetic organization of T4SSs in Hafnia is highly diverse, revealing that the presence of T4SSs is strain-specific but not species-specific. The diversity and strain-specific distribution suggest the likelihood of multiple horizontal transfer events during divergent evolution. As the T4SS phylogenetic analysis generated with shared sequences was not congruent to those obtained with genomic sequences, the distribution of Hafnia T4SS phylogenetic clusters within the SecReT4 database [32] was not assessed further.

\section{Diverse the type $\mathbf{V}$ secretion system}

T5SS is the simplest and most widespread type of secretion pathways [33]. T5SS encodes the translocator and the passenger domains in a single gene or two partner genes $[34,35]$. In this study, based on TXSScan software, T5SSs are divided into three types, designated T5aSS (the classical autotransporter), T5bSS (two partner system), and T5cSS (the trimeric autotransporter) [16]. We found that all 47 Hafnia genomes contain T5SSs (Fig. 4). A total of 370 T5SSs were identified, including 285 T5aSSs, 9 T5bSSs, and 76 T5cSSs. Most of the Hafnia genome contained T5aSSs and T5cSSs. Only 9 strains including 3 alvei and 6 paralvei contained one T5bSS. It is interesting to note that there are notable differences in the numbers of $\mathrm{T} 5 \mathrm{aSSs}$ between alvei $(3.3 \pm 1.8$ per genome) and paralvei ( $9.5 \pm 2.5$ per genome) (Fig. 4$)$. In consideration of T5aSSs can function as enzymes, adhesins, cytotoxins, or mediate bacterial motility, this differential number of T5SSs suggests that the alvei and paralvei differ in their adhesion and evasion abilities [36]. The lager number of T5aSSs may play an important role in the pathogenesis of paralvei, such as the colonization of host cells, biofilm formation, or evasion of the immune system [36]. 


\section{Conversed and diverse the type VI secretion system}

T6SS is present only in gram-negative bacteria and is a phage-tai-spike-like injection machinery [37]. It is thought to contribute for bacterial pathogenesis by the translocation of substrates to the host cells and competition with other bacteria in their niches $[38,39]$. T6SS has not been reported previously in Hafnia. In this study, all Hafnia genomes demonstrated the possession of one or more T6SSs (Fig. 4), suggesting that they may confer some benefit in terms of host colonization and/or pathogenic potential. Although previous studies suggest that strains carrying T6SS may selectively target proteobacterial commensals for the sake of competitive advantage over other potential competitors [40], the function of T6SS in Hafnia remains to be elucidated with further experiments. Based on genetic organizations and homology, the T6SS in Hafnia genomes were divided into 7 subtypes (Fig. 7a). T6SS-1 was conserved in most Hafnia strains, but absent in strains C5 and FDAARGOS_350. Nevertheless, similar to T4SS, the presence of T6SS-2 to -7 was strain-specific but not species-specific. The presence of diverse T4SSs and T6SSs further confirms that HGT is a normal event that occurs in Hafnia.

We constructed an ML tree based on the TssK protein sequences to span the diversity present in Hafnia T6SS (Fig. 7b). In the ML tree, the strains with T6SSs of the same subtype T6SSs form a unique cluster; that is distinct from other subtypes. A similar ML tree generated from TssF is provided in Additional file 5: Figure S1A. This observation reveals the diversity of Hafnia T6SS and the possibility of multiple evolutionary origins. To intuitively understand the diverse origins from an overall perspective, we performed phylogenetic analysis using the shared TssF proteins in the SecReT6 database [41] in combination with the Hafnia T6SS data. As shown in Fig. 7c, the distinct Hafnia T6SSs scatter in different locations, showing high homology to other T6SSs from other species, such as Escherichia coli, Salmonella enterica, Photorhabdus asymbiotica, Enterobacter asburiae, and Citrobacter rodentium. The phylogenetic trees created from the shared TssFs exhibited similar topologies (Additional file 5: Figure S1B). The data indicate that

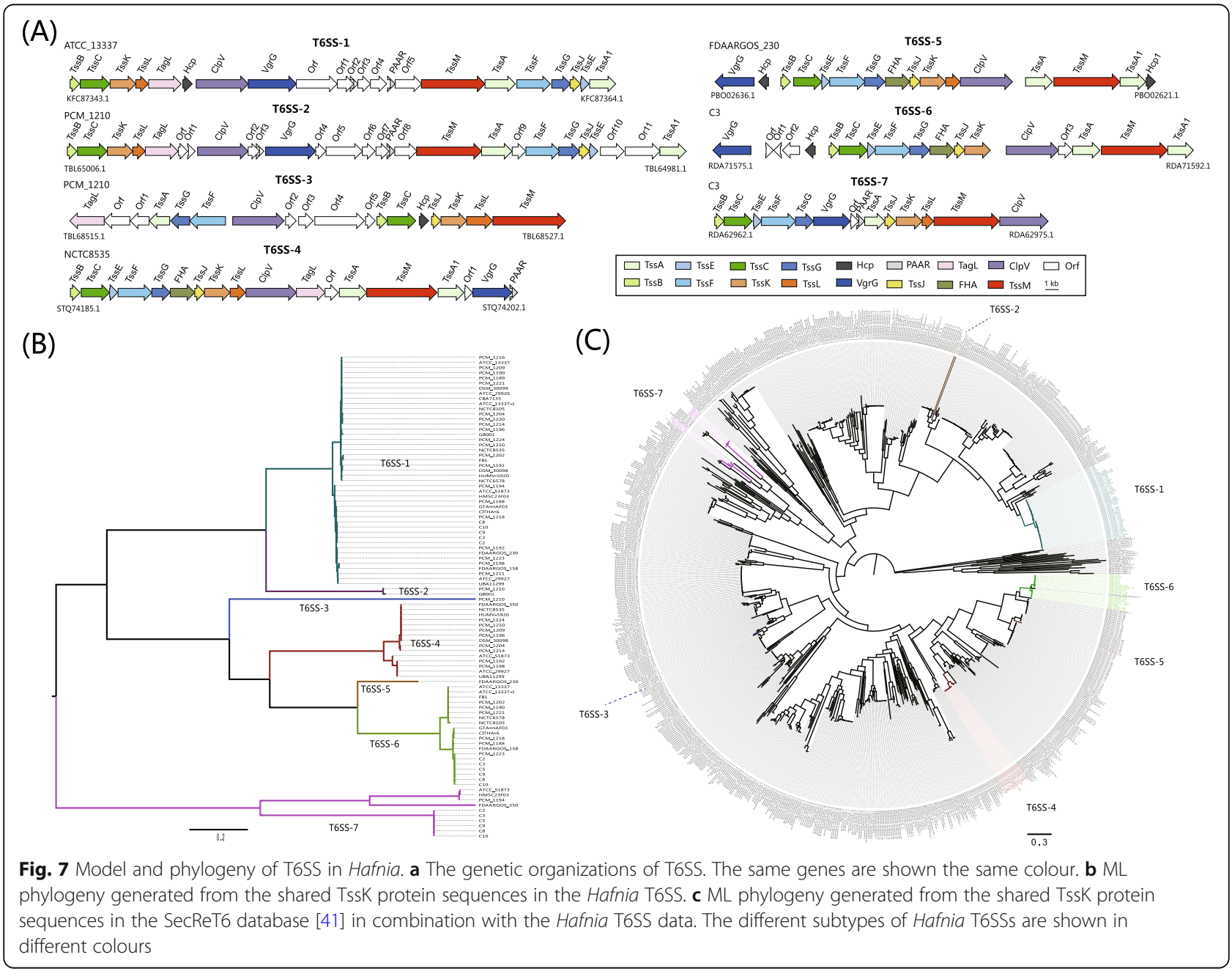


distinct subtypes of Hafnia T6SSs might be horizontally transferred from diverse donor species.

\section{Virulence genotypic profile revealed the pathogenicity of Hafnia}

Mobilome-based elements, such as virulence factors and resistance genes, are very important in pathogenicity and inter-strain variation. In our study, all 47 Hafnia genomes were also locally aligned against the VFDB database, MEGARes, and CARD database to detect virulence factors and resistance genes [42-44]. Virulence factor analysis of the strains examined in this study found that virulence factors represented the greatest numbers of genes among the Hafnia strains (214 virulence factors, Additional file 6: Table S5). Except for previously studied genes related to the macromolecular secretion system, the major virulence factors identified in all strains were associated with adherence (ompA, ilpA, papD, papC, htpB, and csg fimbriae), toxin (hlyA and AHA_3493), iron uptake (chu operon), stress adaptation (katA, clpP, and $\operatorname{sodB}$ ), and efflux pump (farB and $a c r A B$ ). Additionally, alg $U$ (antiphagocytosis), mgtB (magnesium uptake), luxS (quorum sensing), alls (nutritional factor), icl (lipid and fatty acid metabolism) and $r c s B$ (regulation) were identified as common Hafnia virulence factors (Fig. 8).
Several virulence genes were identified as speciesspecific virulence factors that occurred in the majority of strains of one species but were found infrequently in strains of the other species, making them good discriminators between alvei and paralvei (Fig. 8). The alvei-specific genes included $b c f C$, and chuT. The chu operon except for alvei-specific chuT was found to be prevalent in Hafnia strains and was termed the haem transport locus, which appears to be widely distributed among pathogenic E. coli strains [45]. The paralvei-specific genes identified were associated with fimbrial adherence (pefD) and iron uptake (ccmE and $f e p A)$. This differential distribution of virulence factors suggests that alvei and paralvei differ in their adhesion abilities and iron absorption capacities.

Antimicrobial genotypic and phenotypic profiles in Hafnia Fifty resistance genes associated with nine different classes were identified (Fig. 8, Additional file 7: Table S6). All Hafnia genomes contained multiple resistance genes related to aminoglycoside, beta-lactam, bacitracin, cationic antimicrobial peptide, fluoroquinolone, and rifampin. We observed the diversity of acc gene alleles in Hafnia genomes (Fig. 8, Additional file 7: Table S6). Except NCTC8105, all alvei strains contained acc-3 allele. Instead, paralvei strains contained acc-1 (18/21), acc-5 (1/21), and acc-2 (2/21) alleles. This observation




was in agreement with previous study [46]. Similarly, the gyrB alleles related to fluoroquinolone also showed speciesspecific divergence (Res16 and Res17, Additional file 7: Table S6). Except error location of PCM_1191, the ML tree based on $g y r B$ gene sequences separated Hafnia into two species clades with similar topology to core genome tree (Additional file 8: Figure S2). Additionally, we found that many genes encoding efflux pump related to multi-drug resistance are prevalently present in the genome of all Hafnia strains.

All of the 20 Hafnia strains were tested for susceptibility against 21 antimicrobial agents; the results are listed in Additional file 9: Table S7. All strains were uniformly susceptible to ticarcillin-clavulanic acid, cefoperazone-sulbactam, cefepime, imipenem, amikacin, tobramycin, ciprofloxacin, levofloxacin, tigecycline, and trimethoprim-sulfamethoxazole (Additional file 9: Table S7). Almost all strains showed prevalent resistance to amocicillin-clavulanic acid $(100 \% ; n=20)$ and colistin (90\%; $n=18)$. Meanwhile, we observed partial resistance against piperacillin-tazobactam $(25 \% ; n=5)$, ceftriaxone $(35 \% ; n=7)$, ceftazidime $(35 \% ; n=7)$, and ertapenem $(20 \% ; n=4)$. In terms of species susceptibility pattern, some alvei strains were resistant to aztreonam (2/13), meropenem (4/13), and chloramphenicol (2/13), meanwhile, one paralvei strain (PCM_1198) was resistant to doxycycline and minocycline.

\section{Discussion}

Hafnia genus belongs to the family Enterobacteriaceae, and was isolated from the faces of mammals, birds, reptiles, and fish, as well as from soil, water, and foods [47]. It is an opportunistic pathogens that has been implicated in both nosocomial and community-acquired infection. Although it was first identified in 1954, Its taxonomy has remained an unsolved riddle. It is also unclear what the distribution and frequency of these two species are in clinical specimens. Furthermore, the pathogenesis and virulence-related genotype are still not clear. Improvements in next-generation sequencing have resulted in an upsurge of bacterial genome sequences and bioinformatics tools. They have been useful for bacterial taxonomy and provide the opportunity to determine the divergence between species [48]. The genome sequence data of 20 Hafnia strains generated in this study in combined with all 27 publicly available assemblies represented an important contribution to the broader goal of using wholegenome sequences for comparative studies of Hafnia. In this study, phylogenetic and population genetic analysis based on core genomes in combination with wholegenome average nucleotide identity exhibited a reliable delineation of genetic relationships between alvei and paralvei and provided a high-resolution taxonomy of Hafnia. We also identified one previously misclassified as alvei genome, ATCC 51873, which should be labeled as paralvei.

Bacterial genomes are much more variegated than those of eukaryotes. The diverse niches led to the interspecies variations and diverse pathogenesis in strains that could be due to horizontal gene transfer. The diversity of the Hafnia strains is evidenced in the fact that the pan-genome of alvei and paralvei are open. Bared on the COG analysis, both alvei and paralvei contained a larger proportion of core gene families involved in the transcription (category K), energy production (category C), transport and metabolism of carbohydrates and amino acids (categories $G$ and $E$ ). The diverse niche of Hafnia may specifically shape its genome characteristics during ecological adaptation and divergent evolution. Thus, we determined the species-specific core genomes of alvei and paralvei separately. In the KEGG annotation, metabolism and membrane transport were the major functional annotations of these two speciesspecific core genomes. We found three complete pathway modules included the EvgS-EvgA two-component regulation system, methionine transport system, and cellobiose PTS system, are present in the alvei-specific core genomes. The paralvei-specific core genomes included the CreC-CreB two-component regulation system and 2$\mathrm{O}-\alpha$-mannosyl-D-glycerate PTS system. Therefore, the species-specific metabolism-associated gene profiles for alvei and paralvei may reflect the specific nutrient niches in the different host and environments. Moreover, the presence/absence of these species-specific genetic elements would appear to provide a simple and fast approach for identifying alvei and paralvei using PCR.

The Hafnia genomes also harbour the numerous MGEs, and diverse virulence-related genetic profiles that can result from HGT. In particular, the presence of numerous MGEs included CRISPRs, ISs, genomic islands, and prophages may promote an increase in the rate of HGT thus enhancing inter-species divergence. The elements acquired by HGT include metabolic pathways, fitness factors, virulence factors, and resistance genes and can confer fitness, competition, virulence, and antibiotic resistance on the receptor strains [49]. Our observations highlight the potential of Hafnia to engage in robust HGT, which can promote the adaptation of Hafnia to diverse niches and the acquisition of pathogenicity.

Our comparative genomic analysis found that macromolecular secretion systems are widely distributed in the Hafnia genus. These systems include T1SS, flagellum, T3SS, Tad pilus, T4SS, T5SS, and T6SS. Our study revealed the diversity in T4SSs, T5SSs, and T6SSs and the conservation of T1SS, Flagellum 1, and Tad pilus among all strains. It is worth noting that three alvei strains acquired a putative SPI-2 related T3SS from Salmonella 
enterica by HGT event. The observation of this case of pathogenic evolution indicates the potential of the strain to cause severe systemic infection. In addition to possessing a macromolecular secretion system within their genomes, Hafnia strains normally harbour many virulence factors that have been experimentally verified to be important for bacterial pathogenicity [42]. These include the secretion of toxic substances, the production of adherence factors that mediate adhesion and interaction with host cells, metal adsorption, and antibiotic resistance, indicating the ability of Hafnia to damage intestinal epithelial barriers, invade host cells, cause systemic infection, and exhibit multiple drug resistance. Antimicrobial genotypic and phenotypic profiles were also investigated in this study. All Hafnia strains contained several resistance genes related to multiple antimicrobial resistance. Additionally, we found many genes encoding efflux pumps in both alvei and paralvei. These results imply the possibility that the Hafnia strains have acquired multiple resistances to multiple antimicrobials. The susceptibility profile of Hafnia in this study highlighted prevalent resistance to Amocicillin-clavulanic acid and Colistin, partial resistance to Piperacillin-tazobactam, Ceftriaxone, Ceftazidime, and Ertapenem. This finding provides important clues for clinical treatment and antibiotic use.

Our comparative analysis also showed differences in macromolecular systems, virulence, and resistance profiles between alvei and paralvei. The alvei-specific virulence genes included $b c f C$ (fimbrial adherence), and chuT (iron uptake). Most strains of paralvei do not contain Flagellum 2 as a result of an earlier deletion event. The strains of paralvei hold a larger number of T5sSSs, pefD (fimbrial adherence), ccmE and fepA (iron uptake). These variations of virulence-related profiles suggests the divergence in adhesion abilities and iron absorption capacities. The alleles of resistance genes, $a a c$ and $g y r B$ also provided a simple method for identification of both species. We also found that closely related strains of the same species also show genomic diversity. The T3SSs, T4SSs, T5SSs, T6SSs, virulence factors, and resistance genes in Hafnia exhibited high diversity and strain-specific distribution, indicating the likelihood of multiple horizontal transfer events from diverse donor species during divergent evolution. These strain-specific variations indicates that Hafnia strains vary in their pathogenesis.

\section{Conclusion}

In this study, application of whole-genome based phylogeny, population genetic analysis, and ANI analysis provided precise insights into taxonomic relationships among the Hafnia genus. The genome data presented in this study offered important genetic information and a framework for further research. We determined the species-specific genetic profiles that were associated with metabolism, membrane transport, virulence, and antimicrobial resistance, indicating the putative differentiation in niche and pathogenicity between alvei and paralvei. These species-specific genes might serve as a mark for distinguishing alvei and paralvei. Hafnia is an opportunistic pathogen that has been associated with several diseases, including gastroenteritis, bactereamia, and respiratory tract infection. Conserved T1SSs, Flagellum, and Tad pilus and diverse T4SSs, T5SSs, T6SSs, virulence genes, and resistance genes were found in Hafnia pan-genome. We constructed this extensive genomic evaluation of the virulence-related genetic profiles of Hafnia that can help us comprehend the pathogenesis of Hafnia. Clinicians should be aware of this organism and pay attention to Hafnia because it possesses many genetic elements related to virulence and can cause clinically significant infection in an appropriate host.

\section{Methods \\ Bacterial isolates, DNA extraction}

Our 20 Hafnia strains including 7 paralvei and 13 alvei were obtained from the Polish Collection of Microorganisms (PCM) at the Hirszfeld Institute of Immunology and Experimental Therapy, Polish Academy of Sciences (Wroclaw, Poland). All of these strains were found to have different lipopolysaccharides using immunochemical methods. The genetic diversity of O-antigens in our 20 strains has been reported [50]. These strains were grown in liquid medium as previous described [50] and then harvested using centrifugation $(1380 x g$ for $15 \mathrm{~min}$ at $4{ }^{\circ} \mathrm{C}$ ). Bacteria Extraction Kit (CWBIO Co., Ltd., China) were used for DNA extraction from each isolate according to the manufacturer's instructions.

The whole genome of PCM 1220 was sequenced using Pac Bio RS II (Pacific Biosciences), with a depth of approximately 100-fold coverage. The other 19 strains were sequenced using Solexa pair-end sequencing technology (Illumina, Little Chesterford, Essex), with a depth of 90 to 100 fold coverage. For PCM1189 and PCM 1220, a 20-kb library was constructed and end-repaired, and the adaptors were then ligated to generate Single Molecule Real Time (SMRT) bells for circular consensus sequencing. The reads produced with the Pac Bio RS II were de novo assembled using MaSuRCA [51, 52], and those produced with the Solexa pair-end sequencing technology were de novo assembled using Velvet Optimiser v2.2 [53]. The annotation of the genome sequence was conducted using the NCBI Prokaryotic Genome Annotation Pipeline (http://www.ncbi.nlm.nih.gov/genome/annotation_prok). The nucleotide sequences and statistics were submitted to NCBI GenBank (accession numbers listed in Tables). In 
addition, 27 publicly available Hafnia genomes were obtained from NCBI GenBank. All strains used in this study are presented in Additional file 1: Table S1.

\section{Identification of gene orthologous group}

OrthoFinder [54] was used to determine orthologous families of the pan-genome. All protein sequences were compared using a BLASTp all-against-all search with an E-evalue cutoff of $<1 \mathrm{e}-3$. The single-copy core gene, pan gene families and core genome families were extracted from the OrthoFinder output file. Nucleotide sequences of single-copy core genes were extracted according to protein ID.

\section{Phylogenetic analysis}

According to the identification of gene orthologous clusters, a total of 2045 single-copy orthologous core genes shared by per genome. To determine the single nucleotide polymorphisms (SNPs), the nucleotide sequences of single-copy core genes using in core genome phylogenetic analysis were aligned using MAFFT [55] with the default parameter. The SNPs were integrated according to the arrangement of the single-copy genes in complete genome alvei FB1. The phylogeny of SNPs were inferred using the Maximum Likelihood (ML) algorithm in PhyML [56], [with the GTR model of nucleotide substitution and c-distributed rates among sites]. FigTree 1.4.3 (http://tree.bio.ed.ac.uk/ software/figtree) was employed to show the trees. In consideration that homologous recombination caused by horizontal gene transfer occurring in bacterial populations and can confound phylogenetic analysis. We identified and removed putative recombinational regions of the set of SNPs of single-copy core genes, using CloneFrameML [57]. The ML tree of Flagellum 2, TssK, and TssF generated protein sequences which were aligned using the default parameter of MAFFT [55], and were constructed by PhyML using the WAG amino acid substitution model of evolution [56].

\section{Population structure analysis}

The population genetic structure of the Hafnia genus was inferred using the software STRUCTURE 2.3.4 [7] based on SNPs of single-copy core genes, [with $k$ (the number of subpopulation 1-10) and Rep (repeats 5)]. STRUCTURE Harvester [58] assumed $k=2$ subpopulations and correlated allele frequencies, linkage model based on maker distances in base pairs, 10,000-iteration burnin and 10,000 iterations of sampling.

\section{Whole-genome nucleotide identity}

The average nucleotide identity (ANI) and tetramer usage pattern were calculated for the 47 genome dataset using JSpecies 1.2.1 [8], using default parameters. The result was visualized using the pheatmap $\mathrm{R}$ packages.

\section{Pan-genome analysis}

The pan-genome analysis were separately performed on two dataset of 21 paralvei and 26 alvei genomes. According to the Heap's law pan-genome model described in reference [9], the total number of gene clusters is shown for increasing values of the number $N$ of genomes. The curve was a least squares fit of the power law $n=\kappa N^{\gamma}$ to averages. $N$ is the number of genomes, $n$ is the number of core gene clusters, and $\kappa$ and $\gamma$ parameters. The exponent $\gamma>0$ indicates an open pan-genome species.

\section{Species-specific core genome comparison}

To construct accessory genome, we excluded the core gene families and low frequency gene families (shared by less than 10 strain genomes) from pan-genome. To examine the accessory-genome in more detail, we constructed a cluster map for the gene families across all 47 genomes using the heatmap clustering command from the pheatmap R packages (Fig. $2 \mathrm{~d}$ and Additional file 10). We termed the results as "the species-specific core genome" (Additional file 4: Table S4), which represents the set of gene families that are shared across all strains of a species.

\section{Functional category}

We inferred the functional category of the core gene families of alvei and paralvei using the Cluster of Orthologous Group (COG) assignment [59]. The functional annotation of proteins was performed by alignment against the COG database of NCBI using amino acid sequences. Functional analysis of species-specific core genome was done according to the KEGG database.

\section{Identification of MGEs}

The clustered regularly interspaced short palindromic repeats (CRISPRs) were predicted with the CRISPR recognition tool (CRT1.2) [60] with default parameters. Insertion sequences (ISs) were predicted using the IS Finder database. Genomic islands were predicted using IslandViewer. The PHAge search tool (PHAST) was utilized to find the prophages.

\section{Identification of macromolecular secretion systems}

The detection and visualization of Macromolecular systems in Hafnia genus were performed using the program MacSyFinder [22] and TXSScan [16] on the default parameters (http://mobyle.pasteur.fr/cgi-bin/ portal.py\#forms::txsscan.). Furthermore, the T4SS and T6SS were also predicted using SecReT4 [32] and SecReT6 [41] on the default parameters, respectively, 
which annotates and locates the components of T4SS and T6SS on the genome sequences.

\section{The calculation of codon usage and GC-content}

We use CodonW software (available at http://sourceforge.net/projects/codonw) computing statistical parameters of nucleotide composition of gene clusters and genomes such as GC-content and CAI (Codon adaptation index).

\section{Identification of virulence factors and resistance genes}

To identify the virulence factors and resistance genes, the protein sequences of all 47 genomes were aligned using blastp with an E-value cutoff of $<1 \mathrm{e}-6$, identity $>$ $50 \%$, and coverage $>60 \%$ against the dataset from Virulence Factors Database (VFDB), MEGARes, and Comprehensive Antibiotic Resistance Database (CARD) [42-44]. In the final presentation, we removed the virulence factors of previous studied macromolecular secretion system and O-antigen/LPS/Capsule (Fig. 7). The heatmap of distribution of virulence factors and resistance genes were generated by the pheatmap $R$ packages.

\section{Antimicrobial susceptibility testing}

Antimicrobial susceptibility testing was performed for 21 antibiotics, including amocicillin-clavulanic acid, ticarcillin-clavulanic acid, piperacillin-tazobactam, ceftriaxone, ceftazidime, cefoperazone-sulbactam, cefepime, aztreonam, imipenem, meropenem, ertapenem, amikacin, tobramycin, ciprofloxacin, levofloxacin, doxycycline, minocycline, tigecycline, colistin, trimethoprimsulfamethoxazole, and chloramphenicol. MICs were determined by the broth microdilution using dehydrated panels AST-N334 and AST-N335 (Biomerieux, Vitek2) according to standard protocols. Resistance was defined using CLSI criteria. Susceptibility data for our 20 Hafnia strains are shown in Additional file 9: Table S7.

\section{Supplementary information}

Supplementary information accompanies this paper at https://doi.org/10. 1186/s12864-019-6123-1.

Additional file 1: Table S1. Genetic characteristics of strains in the current research.

Additional file 2: Table S2. List of 2045 single-copy genes shared by 47 Hafnia strains.

Additional file 3: Table S3. Average nucleotide identity (ANI)(\%) based on whole genome alignments. ANI values in the species paralvei are in red.

Additional file 4: Table S4. List of alvei and paralvei-specific core genomes with KEGG annotation.

Additional file 5: Figure S2. Maximum likelihood phylogenetic trees generated from the TssF protein sequences in Hafnia T6SS (A) in combination with the SecReT6 database (B).

Additional file 6: Table S5. List of virulence factors in the VFDB database that were identified in Hafnia strains.
Additional file 7: Table S6. List of resistance genes in the CARD and MEGARes database were identified in Hafnia strains.

Additional file 8: Figure S2. Maximum likelihood phylogenetic tree generated from the gyrB gene sequences in Hafnia strains.

Additional file 9: Table S7. Antimicrobial susceptibility profiles of Hafnia.

Additional file 10. The R script command of heatmap clustering for accessory genome analysis.

\section{Abbreviations}

ANI: Average nucleotide identity; BAPS: Bayesian Analysis of Population Structure; CAl: Codon adaptation index; COG: Cluster of Orthologous Group; CRISPR: Clustered regularly interspaced short palindromic repeats;

CRISPR: The clustered regularly interspaced short palindromic repeats; $H$. alvei: Hafnia alvei; H. paralvei: Hafnia paralvei; HGT: Horizontal gene transfer; IS: Insertion sequence; KEGG: Kyoto Encyclopedia of Genes and Genomes; MGEs: Mobile genetic elements; ML: Maximum Likelihood; NCBI: National Center of Biotechnology Information; ORF: Open reading frame; PHAST: PHAge search tool; T1SS-T6SS: Type I - VI secretion; Tad: Tight adherence; VFDB: Virulence Factors Database

\section{Acknowledgments}

The authors thank prof. Andrzej Gamian and dr Stefan Rowinski of the Hirszfeld Institute of Immunology and Experimental Therapy, Wroclaw, Poland for their assistance with obtaining and handling of the bacterial strains from the Polish Collection of Microorganisms.

\section{Author's contributions}

$\mathrm{DH}, \mathrm{BL}$, and $\mathrm{ZY}$ designed the research. $C Y, Y D$, and $Z Y$ performed the laboratory work and bioinformatics analyses. YD and ZY wrote the manuscript and $B L$ revised it. PY, CQ, YW, and SZ contributed with conceptual and technical expertise in bioinformatics and laboratory work and advices in manuscript. DH and $B L$ made substantial contributions to conception, design of fundamental questions, analysis and interpretation of data and review or revision of manuscript. All authors read and approved the final manuscript.

\section{Funding}

This work was funded by the National Key Programs for Infectious Diseases of China (No. 2017ZX10104002-001-006 to BL) and the National Natural Science Foundation of China (No. 81471904, 81772148, and 81611530714 to $\mathrm{BL})$. The funding bodies did not exert influence on the design of the study and collection, analysis, and interpretation of data and in writing the manuscript. The opinions expressed in this paper are those of the authors.

\section{Availability of data and materials}

The genome sequencing data of our 20 Hafnia strains has been deposited at NCBI GenBank under the BioProject accession no. PRJNA523071 and the genome BioSample accession no. (SAMN10963441 - SAMN10963460). All genome sequences and annotations referenced in the manuscript are publically available on the GenBank Database at the accession number provided (Additional file 1: Tale S1).

Ethics approval and consent to participate Not applicable.

\section{Consent for publication}

Not applicable.

\section{Competing interests}

The authors declare that they have no competing interests.

\section{Author details}

'Key Laboratory of Molecular Microbiology and Technology of the Ministry of Education, TEDA College, Nankai University, Tianjin, People's Republic of China. ${ }^{2}$ TEDA institute of Biological Sciences and Biotechnology, Nankai University, Tianjin, People's Republic of China. ${ }^{3}$ Tianjin Key Laboratory of Microbial Functional Genomeics, TEDA college, Nankai university, Tianjin, People's Republic of China. 
Received: 9 April 2019 Accepted: 20 September 2019

Published online: 23 October 2019

\section{References}

1. Janda JM, Abbott SL, Bystrom S, Probert WS. Identification of two distinct hybridization groups in the genus Hafnia by 165 rRNA gene sequencing and phenotypic methods. J Clin Microbiol. 2005:43:3320-3.

2. Huys G, Cnockaert M, Abbott SL, Janda JM, Vandamme P. Hafnia paralvei sp. nov., formerly known as Hafnia alvei hybridization group 2. Int J Syst Evol Microbiol. 2010;60:1725-8.

3. Gelev I, Gelev E, Steigerwalt AG, Carter GP, Brenner DJ. Identification of the bacterium associated with haemorrhagic septicaemia in rainbow trout as Hafnia alvei. Res Microbiol. 1990;141:573-6.

4. Janda JM, Abbott SL. The genus Hafnia: from soup to nuts. Clin Microbiol Rev. 2006:19:12-28.

5. Litrenta J, Oetgen M. Hafnia alvei: a new pathogen in open fractures. Trauma Case Rep. 2017:8:41-5.

6. Cardile AP, Forbes D, Cirigliano V, Stout B, Das NP, Hsue G. Hafnia alvel pyelonephritis in a renal transplant recipient: case report and review of an under-recognized nosocomial pathogen. Transpl Infect Dis. 2011;13:407-10.

7. Falush D, Stephens M, Pritchard JK. Inference of population structure using multilocus genotype data: dominant markers and null alleles. Mol Ecol Notes. 2007;7:574-8.

8. Richter M, Rosselló-Móra R. Shifting the genomic gold standard for the prokaryotic species definition. Proc Natl Acad Sci U S A. 2009;106:19126-31.

9. Tettelin H, Riley D, Cattuto CMD. Comparative genomics : the bacterial pangenome. Curr Opin Microbiol. 2008;11:472-7.

10. Eguchi $Y$, Oshima $T$, Mori H, Aono R, Yamamoto K, Ishihama A, et al. Transcriptional regulation of drug efflux genes by EvgAS, two-component system in Escherichia coli. Microbiology. 2003;149:2819-28.

11. Hetzler SSA. Establishment of cellobiose utilization for lipid production in Rhodococcus opacus PD630. Appl Environ Microbiol. 2013;79:3122-5.

12. Sekar R, Shin HD, Chen R. Engineering Escherichia coli cells for cellobiose assimilation through a phosphorolytic mechanism. Appl Environ Microbiol. 2012;78:1611-4

13. Merlin C, Gardiner G, Durand S, Masters M. The Escherichia coli metD locus encodes an ABC transporter which includes Abc (MetN), YaeE (Metl), and YaeC (MetQ). J Bacteriol. 2002;184:5513-7.

14. Godoy MS, Nikel PI, Cabrera Gomez JG, Julia PM. The CreC regulator of Escherichia coli, a new target for metabolic manipulations. Appl Environ Microbiol. 2016:82:244-54.

15. Rock CO, Dippel R, Lu Y-J, Boos W, Sampaio M-M, Schlegel A, et al. Phosphotransferase-mediated transport of the osmolyte 2-O-alphamannosyl-D-glycerate in Escherichia coli occurs by the product of the $\mathrm{mngA}$ ( hrs $A$ ) gene and is regulated by the $m n g R$ (farR) gene product acting as repressor. J Biol Chem. 2004;279:5537-48.

16. Abby SS, Rocha EPC. Identification of protein secretion systems in bacterial genomes using MacSyFinder. Methods Mol Biol. 2017;1615:1-21.

17. Raymond B, Young JC, Pallett M, Endres RG, Clements A, Frankel G. Subversion of trafficking, apoptosis, and innate immunity by type III secretion system effectors. Trends Microbiol. 2013;21:430-41.

18. Kanonenberg K, Schwarz CKW, Schmitt L. Type I secretion systems - a story of appendices. Res Microbiol. 2013;164:596-604.

19. Economou A, Christie PJ, Fernandez RC, Palmer T, Plano GV, Pugsley AP. Secretion by numbers: protein traffic in prokaryotes. Mol Microbiol. 2006;62: 308-19.

20. Bleves S, Viarre V, Salacha R, Michel GPF, Filloux A, Voulhoux R. Protein secretion systems in Pseudomonas aeruginosa: a wealth of pathogenic weapons. Int J Med Microbiol. 2010;300:534-43.

21. Dalbey RE, Kuhn A. Protein traffic in gram-negative bacteria - how exported and secreted proteins find their way. FEMS Microbiol Rev. 2012;36:1023-45.

22. Touchon M, Rocha EPC, Abby SS, Ne B. MacSyFinder: a program to mine genomes for molecular systems with an application to CRISPR-Cas systems. PLoS One. 2014;9:e110726.

23. Wells TJ, Henderson IR. Type 1 and 5 secretion systems and associated toxins. Second Edi. Escherichia coli Pathotypes Princ. Pathog. Second Ed. Elsevier; 2013. p. 499-532.

24. Akatsuka H, Kawai E, Omori K, Shibatani T. The three genes lipB, lipC, and lipD involved in the extracellular secretion of the Serratia marcescens lipase which lacks an N-terminal signal peptide. J Bacteriol. 1995;177:6381-9.
25. Diepold A, Armitage JP. Type III secretion systems: the bacterial flagellum and the injectisome. Philos Trans R Soc B Biol Sci. 2015;370. https://doi.org/ 10.1098/rstb.2015.0020.

26. Cornelis GR. The type III secretion injectisome. Nat Rev Microbiol. 2006:4: 811-25.

27. Sabbagh SC, Forest CG, Lepage C, Leclerc JM, Daigle F. So similar, yet so different: uncovering distinctive features in the genomes of Salmonella enterica serovars Typhimurium and Typhi. FEMS Microbiol Lett. 2010;305:113.

28. Hensel M. Salmonella pathogenicity island 2. Mol Microbiol. 2000;36:101523.

29. Mignolet J, Panis G, Viollier PH. More than a tad: spatiotemporal control of Caulobacter pili. Curr Opin Microbiol. 2018;42:79-86.

30. Tomich M, Planet PJ, Figurski DH. The tad locus: postcards from the widespread colonization island. Nat Rev Microbiol. 2007:5:363-75.

31. Guglielmini J, De La Cruz F, Rocha EPC. Evolution of conjugation and type IV secretion systems. Mol Biol Evol. 2013;30:315-31.

32. Liu L, Tai C, Bi D, Ou H-Y, Rajakumar K, Deng Z. SecReT4: a web-based bacterial type IV secretion system resource. Nucleic Acids Res. 2012;41: D660-5.

33. Dautin N, Bernstein HD. Protein secretion in gram-negative Bacteria via the autotransporter pathway. Annu Rev Microbiol. 2007;61:89-112.

34. Van Ulsen P, S ur R, WSP J, Daleke-Schermerhorn MH, Luirink J. Type V secretion: From biogenesis to biotechnology. Biochim Biophys Acta, Mol Cell Res. 1843;2014:1592-611.

35. Bönemann G, Pietrosiuk A, Diemand A, Zentgraf H, Mogk A. Remodelling of VipANipB tubules by ClpV-mediated threading is crucial for type VI protein secretion. EMBO J. 2009;28:315-25.

36. Grijpstra J, Arenas J, Rutten L, Tommassen J. Autotransporter secretion: varying on a theme. Res Microbiol. 2013;164:562-82.

37. Coulthurst SJ. The type VI secretion system - a widespread and versatile cell targeting system. Res Microbiol. 2013;164:640-54.

38. Records AR. The type VI secretion system: a multipurpose delivery system with a phage-like machinery. Mol Plant-Microbe Interact. 2011;24:751-7.

39. Cianfanelli FR, Monlezun L, Coulthurst SJ. Aim, load, fire: the type VI secretion system, a bacterial Nanoweapon. Trends Microbiol. 2016;24:51-62.

40. Russell AB, Peterson SB, Mougous JD. Type VI secretion system effectors: poisons with a purpose. Nat Rev Microbiol. 2014;12:137-48.

41. Li J, Yao Y, Xu HH, Hao L, Deng Z, Rajakumar K, et al. SecReT6: a web-based resource for type $\mathrm{VI}$ secretion systems found in bacteria. Environ Microbiol. 2015;17:2196-202

42. Liu B, Zheng D, Jin Q, Chen L, Yang J. VFDB 2019: a comparative pathogenomic platform with an interactive web interface. Nucleic Acids Res. 2019;47:D687-92.

43. Lakin SM, Dean C, Noyes NR, Dettenwanger A, Ross S, Doster E, et al. MEGARes : an antimicrobial resistance database for high throughput sequencing. Nucleic Acids Res. 2017:45:574-80.

44. Jia B, Raphenya AR, Alcock B, Waglechner N, Guo P, Tsang KK, et al. CARD 2017: expansion and model-centric curation of the comprehensive antibiotic resistance database. Nucleic Acids Res. 2017;45:D566-73.

45. Torres AG, Payne SM. Haem iron-transport system in enterohaemorrhagic Escherichia coli O157:H7. Mol Microbiol. 1997;23:825-33.

46. Abbott SL, Green N, Moler S, Wainwright K, Janda JM, Tran RK. Clinical and laboratory diagnostic characteristics and cytotoxigenic potential of Hafnia alvei and Hafnia paralvei strains. J Clin Microbiol. 2011;49:3122-6.

47. Choi H-J, Kwon J, Choi J-S, Kang J, Kim JS, Kim YB, et al. Complete genome sequence of a commensal bacterium, Hafnia alvei CBA7124, isolated from human feces. Gut Pathog. 2017;9:41.

48. Kaas RS, Friis C, Ussery DW, Aarestrup FM. Estimating variation within the genes and inferring the phylogeny of 186 sequenced diverse Escherichia coli genomes. BMC Genomics. 2012;13:577.

49. Stecher B, Maier L, Hardt WD. "Blooming" in the gut: how dysbiosis might contribute to pathogen evolution. Nat Rev Microbiol. 2013;11:277-84.

50. Duan Z, Niedziela T, Lugowski C, Cao B, Wang T, Xu L, et al. Genetic diversity of O-antigens in Hafnia alvei and the development of a suspension array for serotype detection. PLoS One. 2016;11:e0155115.

51. Kuang Y, Hu M, Wu Q. A new rhesus macaque assembly and annotation for next-generation sequencing analyses. Biol Direct. 2014;14:1.

52. Tallon LJ, Liu X, Bennuru S, Chibucos MC, Godinez A, Ott S, et al. Single molecule sequencing and genome assembly of a clinical specimen of Loa loa, the causative agent of loiasis. BMC Genomics. 2014;15:788. 
53. Zerbino DR, Birney E. Velvet: algorithms for de novo short read assembly using de Bruijn graphs. Genome Res. 2008;18:821-9.

54. Emms DM, Kelly S. OrthoFinder: solving fundamental biases in whole genome comparisons dramatically improves orthogroup inference accuracy. Genome Biol. 2015;16:157.

55. Katoh K, Standley DM. MAFFT Multiple Sequence Alignment Software Version 7 : Improvements in Performance and Usability Article Fast Track. Mol Biol Evol. 2013;30:772-80.

56. Guindon S, Gascuel O. A simple, fast, and accurate algorithm to estimate large phylogenies by maximum likelihood. Syst Biol. 2003;52:696-704.

57. Didelot X, Wilson DJ. ClonalFrameML: efficient inference of recombination in whole bacterial genomes. PLoS Comput Biol. 2015;11:e1004041.

58. Earl DA, von Holdt BM. Structure harvester: a website and program for visualizing STRUCTURE output and implementing the Evanno method. Conserv Genet Resour. 2012:4:359-61.

59. Galperin MY, Makarova KS, Wolf YI, Koonin EV. Expanded microbial genome coverage and improved protein family annotation in the COG database. Nucleic Acids Res. 2015;43:D261-9.

60. Bland C, Ramsey TL, Sabree F, Lowe M, Brown K, Kyrpides NC, et al. CRISPR recognition tool (CRT): a tool for automatic detection of clustered regularly interspaced palindromic repeats. BMC Bioinformatics. 2007:8:209.

\section{Publisher's Note}

Springer Nature remains neutral with regard to jurisdictional claims in published maps and institutional affiliations.

Ready to submit your research? Choose BMC and benefit from:

- fast, convenient online submission

- thorough peer review by experienced researchers in your field

- rapid publication on acceptance

- support for research data, including large and complex data types

- gold Open Access which fosters wider collaboration and increased citations

- maximum visibility for your research: over $100 \mathrm{M}$ website views per year

At $\mathrm{BMC}$, research is always in progress.

Learn more biomedcentral.com/submissions 